\title{
The Nursing Outcomes Classification
}

\author{
Classificação dos Resultados das Intervenções de Enfermagem
}

Clasificación de los resultados de las intervenciones de Enfermería

\section{Sue Ann Moorhead ${ }^{1}$}

\section{INTRODUCTION}

The research to develop the Nursing Outcomes Classification (NOC) began with the formation of the outcomes research team in 1991. This work grew from previous language development focused on patient problems or nursing diagnoses by the North American Nursing Diagnosis Association and the development of nursing interventions by the Nursing Interventions Classification (NIC) research team at Iowa. Outcome terminology was needed to complete the clinical reasoning model identified as part of the NIC research ${ }^{(1)}$. Figure 1 depicts this model and its relationship to clinical reasoning.

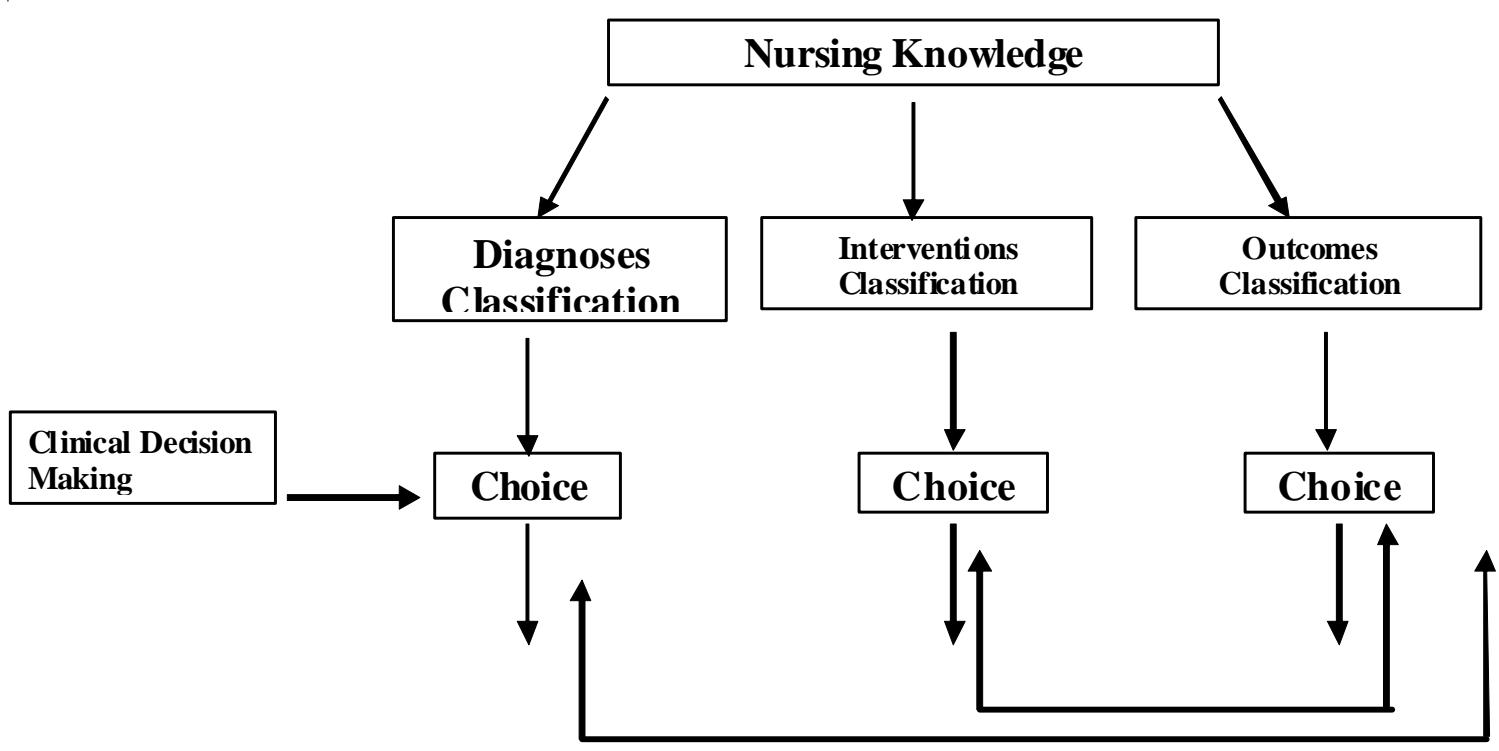

Figure 1- Relationship of Nursing Knowledge Classifications to the Nurse's Clinical Decision Making(2)

This model is based on the nursing process and helps the nurse focus on identifying the three knowledge domains of nursing focused on diagnoses, interventions and outcomes. Clinical decisions about patient care are made considering the patient problem, possible outcomes, and the interventions that will improve or eliminate the problems faced by

1 Associate Professor Iowa Board of Nursing. The University of Iowa, EUA. 
the patient. This is not a linear process but is based on the reflection of the nurse on the patient's responses to treatment. Alterations in the plan are made as vaiations occur in the expected outcomes.

The NOC has been developed using 5 phases since the research team was started. Phase 1 focused on pilot work to test methodologies for developing outcomes from 1992 to 1993. This phase of the research was funded by Sigma Theta Tau International. Phase 2 was devoted to the construction of the first set of outcomes (1993-1996) and the publication of the first edition of the classification ${ }^{(3)}$. Phase 3 (1996-1997) focused on the construction of a taxonomy of the classification and the beginning of clinical testing of the outcomes. This expanded the classification from an alphabetical list to a structure to group related concepts in a three-tiered structure and was published in the second edition $^{(4)}$. Phase 4 (1998-2002) was dedicated to evaluation of the measurement scales in NOC in a variety of settings. Phase 5 has focused on the refinement and clinical use of the outcomes and has been ongoing since 1997. Funding for phases 2 through 5 was obtained from the National Institutes of Health, National Institute of Nursing. The last ten years has been devoted to refining the outcomes previously published, the development of new outcomes, assisting practicing nurses to implement NOC in their practice, and assisting educators to include NOC in curriculum revisions.

\section{RESEARCH METHODS USED TO DEVELOP NOC}

Multiple research methods have been used in the development of NOC. An inductive approach was used to develop the outcomes based on current practice and research. Concept analysis and research team review were used in the construction of the outcomes. Questionnaire surveys of expert nurses were used to assess the content validity and nursing sensitivity of the outcomes. The taxonomy was constructed using similarity/dis-similarity analysis and hierarchical clustering techniques. Feedback from clinical test sites and other sites implementing NOC have been used to identify new outcomes for development and refine current outcomes. Inter-rater reliability and criterion measures were used to evaluate the reliability, validity, and sensitivity of the outcome measures in clinical sites. This data was included in the third edition of NOC ${ }^{(5)}$. Expert review continues to be used as new outcomes are developed.

\section{THE BASICS OF USING NOC AND MEASURING OUTCOMES}

The fourth edition of NOC published in $2008^{(6)}$ contains 385 outcomes. Each outcome consists of a definition, set of indicators, measurement scale(s) and supporting references. Outcomes can be focused on the patient or caregiver, the family as the unit of analysis, or on a community or population. "A nursing-sensitive patient outcome is an individual, family, or community stat, behavior or perception that is measured along a continuum in response to nursing interventions"(()). The outcomes are developed as variable concepts that can be measured along a continuum using a measurement scale. A 5-point Likert scale is used with all outcomes. The "five" represents that best possible score on the outcome and the "one" represents the worst possible score. A five-point scale allows for an adequate number of responses to demonstrate variability in the outcome state, perception, or behavior of interest to the nurse. The outcome is measured prior to nursing interventions to establish a baseline score. The outcome is then rated again post intervention to determine a change score for the outcome. Outcome change scores can be positive (the outcome rating increase), negative (the outcome rating decreased) or there can be no change (the outcome stayed the same).

We advocate the use of a "reference person" when measuring outcomes. A reference person is an individual of the same age and gender. For example a female patient who is 30 years old should be compared to a healthy woman age 30. This is important to keep the rating of " 5 " as the score for a healthy person across settings and populations. This does mean that patients with chronic illness that impact the outcomes may not be able to achieve a " 5 " rating due to their chronic illness. We do not want the top rating to be undermined by conditions that reflect the best state of the patients the nurse work. This is especially true for patients with medical conditions such as congestive heart failure, renal failure, dementia, or other serious conditions.

The NOC outcomes are at a higher level of abstraction than the goals nurses have typically included in care plans. The indicators provide examples of more specific states, perceptions, or behaviors usually seen as indicative of the outcome. The indicators can also be rated as individual items to identify key areas to target with the selected nursing interventions. This may be especially helpful as nurses learn to use NOC. These indicators are not summed to create an overall score because we know some indicators are more important for determining the outcome than others. Further research is needed to identify the key indicators for each outcome. These may vary by patient population, setting, or specialty practice.

There are currently fourteen scales used in the $4^{\text {th }}$ edition to measure outcomes. In this edition there are 298 
outcomes that use only one scale. Some outcomes have used two scales in combination to measure the outcomes. In this case there is a primary scale used to determine change scores. The most commonly used scales are Severely compromised to Not compromised (measures a patient state); Never demonstrated to Consistently Demonstrated (measures a patient behavior); No knowledge to Extensive knowledge (to measure patient knowledge) and Not at all satisfied to Completely satisfied (to measure a perception). Nurses determine the interval for outcome measurement based on clinical judgment as to when the effects of interventions need to be assessed. This may vary across patient populations and settings. Organization policies may also affect measurement intervals. At minimum outcomes should be measured on admission for a baseline, at discharge or transfer to another unit, and when there is a significant change in status for the outcome.

We have attempted to link knowledge outcomes with corresponding outcomes focused on behavior. We think these are important outcomes for nurses to determine the behavioral outcomes associated with the teaching interventions they perform. For example, the outcome Knowledge: Asthma is associated with the following behavioral outcomes: Asthma Self-Management, Energy Conservation, Health Seeking Behavior, Symptom Control, and Risk Control: Infectious Process to name a few. These behavioral outcomes reflect the effect of acquiring knowledge to better control this disease. These behavioral outcomes will help document the value of nursing care for patients with chronic illness and the importance of interventions focused on teaching the patients about their health conditions.

\section{RESOURCES FOR USING NOC}

The Outcome-Present State Test (OPT) Model developed by Pesut and Herman ${ }^{(7)}$ is an excellent model to help nurses with clinical reasoning in complex patient situations. This model is supportive of the original model of knowledge development and clinical reasoning developed by the NIC team and focuses on clinical reasoning beginning with the patient's story. The key components of the model are cue logic using clinical reasoning webs. These webs help the nurse identify the capstone issue, or the priority problem to focus care planning. The model uses reflection, framing, testing and decision-making process to compare the present state with future state following the chosen intervention. NOC outcomes provide the concepts for comparing present state and future state component of the model and can be enhanced by using NANDA diagnoses and NIC interventions as concepts for the other knowledge domains.

Another resource for nurses using NANDA diagnoses, NIC Interventions and NOC outcomes is the book devoted to combing the content of all three knowledge bases. This "linkage book" identified several NOC outcomes and associated NIC interventions for all NANDA diagnoses published to date ${ }^{(8)}$. There have been two edition of this text to date ${ }^{(8-9)}$. The current edition focuses on all diagnoses published in the 2006-2008 classification of NANDA International ${ }^{(10)}$. This book is a useful tool for nurses and students learning to use all three languages to describe the care they provide to patients, regardless of population or setting. Case studies in this book are helpful for educators to use in their courses.

\section{CONCLUSION}

The use of standardized languages is becoming more prevalent in practice and education because nursing textbooks are including the use the nursing languages such as NOC and staff nurses and faculty are becoming more familiar with them. The validation of standardized languages and their use in nursing practice and research is still in its infancy, but is important to the nursing profession as the implementation of evidence-based practice and the electronic medical record provide more opportunities to test the use of standardized languages in practice settings. For standardized, nursing languages such as NOC to become consistently used, it requires consistent incorporation of the languages in nursing practice, education, and research. Models that support clinical decision making are important tools for assisting nurses to improve the care they provide to patients and their families.

\section{REFERENCES}

1. McCloskey JC, Bulechek GM, editors. Nursing interventions classification (NIC): Iowa Intervention Project. St. Louis: Mosby Year Book; 1992.

2. McCloskey JC, Bulechek GM, editors. Nursing interventions classification (NIC): Iowa Intervention Project. 2nd ed. St. Louis: Mosby; c1996. p.6

3. Johnson M, Maas M, editors. Nursing outcomes classification (NOC): Iowa Outcomes Project. St. Louis: Mosby; c1997.

4. Johnson M, Maas M, Moorhead S, editors. Nursing outcomes classification (NOC). Iowa Outcomes Project: 2nd ed. St. Louis: Mosby; 2000.

5. Moorhead S, Johnson M, Maas M, editors. Nursing outcomes classification (NOC). 3rd ed. St. Louis: Mosby; 
2004.

6. Moorhead S, Johnson M, Maas M, Swanson E, editors. Nursing outcomes classification (NOC). 4th ed. St. Louis: Mosby; 2008.

7. Pesut DJ, Herman J. Clinical reasoning: the art and science of critical \& creative thinking. Boston: Delmar; 1999.

8. Johnson M, Bulechek G, Butcher H, Maas M, McCloskey Dochterman J, Moorhead S, Swanson L. Nursing diagnoses, outcomes and interventions: NANDA, NOC, and NIC linkages. St. Louis: Mosby; 2006.

9. Johnson M, Bulechek G, Maas M, McCloskey Dochterman J, Moorhead S, editors. Nursing diagnoses, outcomes and interventions: NANDA, NOC, and NIC linkages. St. Louis: Mosby; c2001.

10. North American Nursing Diagnosis Association. NANDA nursing diagnoses: definitions \& classification, 2006-2008. 7th ed. Philadelphia: North American Nursing Diagnosis Association; 2006. 